\title{
TRANSPARENCIA Y ACCESO A LA INFORMACIÓN EN EL SITIO DEL DEFENSOR DEL PUEBLO: UN ESTUDIO LONGITUDINAL
}

\author{
Aires José Rover ${ }^{1}$ \\ Paloma Maria Santos ${ }^{2}$
}

\begin{abstract}
Resumen
Los sitios de gobierno deben ser cada vez más abiertos, acogedores y receptivos al control social, con el fin de que el ciudadano participe más en el proceso de toma de decisión. Así, deben presentar contenidos específicos para su público y permitir la construcción y el intercambio de conocimiento. Siendo el acceso y la publicidad de información condiciones esenciales al establecimiento del Estado Democrático de Derecho, este artículo busca verificar si las prerrogativas de la Ley española 19/2013, de Transparencia, Acceso a la Información Pública y Buen Gobierno, están siendo atendidas por la institución del Defensor del Pueblo. Sus aspectos más importantes, referentes a la exigencia de publicidad activa en materia institucional, organizativa, de relevancia jurídica, económica, presupuestaria y estadística, el reconocimiento del derecho de acceso a la información pública y la creación de un procedimiento para el ejercicio del mismo, fueron traducidos en 30 preguntas, estructuradas bajo la forma de un cuestionario creado a partir de la herramienta Survey Monkey. La evaluación mostra que los procedimientos establecidos por la Ley, con el fin de garantizar el acceso a la información, aún no son integralmente atendidos por la Institución Defensor del Pueblo.
\end{abstract}

Palabras clave: Estudio longitudinal; Sitios de gobierno; Transparencia Pública; Acceso a la Información; Estado Democrático de Derecho.

\section{INTRODUCCIÓN}

Varios son los estudios que tienen como objetivo medir la apertura del gobierno y la disponibilidad del acceso a la información a disposición del público (CORONEL, 2012). Esto es reflejo, en gran parte, del creciente interés mundial por la utilidad de la transparencia como una herramienta catalizadora de la reforma, y con la aparición, desde los años 1990, de un movimiento hacia la información global.

Con el surgimiento de los sitios web, no hay más cómo justificar la indisponibilidad de medios para

\footnotetext{
${ }^{1}$ Professor Adjunto do Programa de Pós-Graduação em Direito e do Programa de Pós-Graduação em Engenharia e Gestão do Conhecimento. E-mail: aires.rover@gmail.com

${ }^{2}$ Pesquisadora Pós-doutoranda no Programa de Pós-Graduação em Engenharia e Gestão do Conhecimento da Universidade Federal de Santa Catarina - UFSC. Doutora e Mestre em Engenharia e Gestão do Conhecimento pela UFSC. E-mail: santos.paloma@gmail.com
} 
fornecer la información, ya que, según Silva (2009), los sitios acaban sirviendo como un repositorio, accesible a personas espacialmente distantes, a cualquier momento.

Los sitios soportan la organización y la distribución de la información y del conocimiento dentro de una organización. Son capaces de realizar la conversión del conocimiento implícito para explícito, la medida en que integran informaciones de diferentes fuentes en un único punto de acceso y las presentan de una manera coherente para sus usuarios (LINDVALL; RUS; SINHA, 2003; AL-MUDIMIGH; ULLAH, 2011).

Considerados como uno de los canales más importantes para la interacción entre gobierno y ciudadanos, los sitios de gobierno deben ser cada vez más abiertos, acogedores y receptivos a la participación y al control social, con el fin de insertar al ciudadano en el proceso de toma de decisión.

De esta forma, deben presentar contenidos específicos para su público y permitir la construcción y el intercambio de conocimiento, la medida en que disponen de herramientas para comunicación entre quien produce y quien consume la información (SANTOS, 2014).

Siendo el acceso y la publicidad de información condiciones esenciales para el establecimiento del Estado Democrático de Derecho, este artículo busca realizar un estudio longitudinal para verificar si las prerrogativas de la Ley española 19/2013, de 9 de diciembre, de Transparencia, Acceso a la Información Pública y Buen Gobierno, están siendo atendidas por la institución del Defensor del Pueblo.

Para ello, además de esta introducción, se discute en la sección 2 la importancia de la transparencia en el sector público. La sección 3 trae las legislaciones sobre la materia vigentes en España. En la sección 4 se tiene la metodología utilizada. La sección 5 aborda el análisis de los datos. En la sección 6, la síntesis de los resultados, y, finalmente, en la sección 7, las consideraciones finales y las oportunidades de trabajos futuros.

\section{TRANSPARENCIA EN EL SECTOR PÚBLICO}

La búsqueda por la transparencia en la gestión pública es una condición necesaria, aunque no suficiente, para el establecimiento de un régimen democrático (MARQUES, 2004).

Aunque la transparencia parezca una cuestión simple, de flujo abierto de información, no lo es. La transparencia requiere de condiciones sociales, culturales y económicas para que se establezca y depende fundamentalmente del acceso a informaciones que están bajo la posesión de las autoridades, que creemos decir la verdad - aunque se sabe que esa premisa ni siempre es cierta (HOLZNER; HOLZNER, 2006).

La máquina pública, como observa Torres (2004), sufre constantemente con la corrupción, y, de una forma u otra, la transparencia y la apertura de informaciones son componentes que pueden ayudar a atacar ese 
problema, así como a mejorar las acciones estatales.

Además de reforzar la actuación democrática de los gobiernos, la transparencia emerge de la necesidad de atención a los principios constitucionales que rigen la organización y el funcionamiento del Estado, así como de la urgencia de modernización impuesta al modelo de gestión pública (AMORIM, 2012).

De acuerdo con la OCDE (2009), el desarrollo transparente y participativo de las políticas se presenta, en la mayoría de las veces, como un medio para mejorar el desempeño de la democracia, ya que puede fortalecer la transparencia y la rendición de cuentas de los gobiernos, fomentando la participación pública y el desarrollo del sentido cívico.

Se resalta, sin embargo, que, para la plena realización de la transparencia, no basta que el gobierno ofrezca las informaciones concernientes a lo que la ley obliga, sino también que disponga de mecanismos que posibiliten al ciudadano ejercer un efectivo control social.

La visibilidad y transparencia de las acciones del gobierno permiten, más allá del control de los actos, la percepción por parte de las personas sobre las actitudes (i)lícitas de sus representantes (BOBBIO, 2002).

\section{LEGISLACIÓN ESPAÑOLA SOBRE TRANSPARENCIA Y ACCESO A LA INFORMACIÓN PÚBLICA}

Las administraciones públicas en todo el mundo viven un momento muy especial en lo que se refiere a la apertura de datos y al acceso a la información.

En Europa y, en particular, en España, algunos movimientos merecen ser destacados:

1. Ley $37 / 2007$, de 16 de noviembre, sobre reutilización de la información del sector público: tiene como objetivo armonizar la explotación de la información producida o custodiada por las Administraciones y organismos del sector público al objeto de contribuir al crecimiento económico y la creación de productos y servicios, sirviendo como elemento de transparencia y guía para la participación democrática, reforzando la eficacia de su uso por parte de los ciudadanos y de las empresas. Aunque se prevea que la decisión de autorizar la reutilización sea del sector público, la ley pretende estimular la puesta a disposición de la información por medios electrónicos. (ESPAÑA, 2007).

2. Ley 19/2013, de 9 de diciembre, de Transparencia, Acceso a la Información Pública y Buen Gobierno: tiene como objetivo ampliar y reforzar las obligaciones de publicidad activa en torno a las actividades públicas, regular y garantizar el derecho de acceso a la información y establecer las obligaciones de buen gobierno que deben cumplir los responsables públicos en distintos ámbitos. Con este triple alcance, la 
ley supone un importante avance en dirección a la consolidación de la democracia, pasando de la cultura del secreto a la del acceso, siendo que en ese escenario la información es la regla y el sigilo la excepción. La ley se aplica a todas las administraciones públicas y a todo el sector público estatal. (ESPAÑA, 2013).

3. Ley 39/2015, de 1 de octubre, del Procedimiento Administrativo Común de las Administraciones Públicas: tiene como objetivo regular los requisitos de validez y eficacia de los procedimientos administrativos tramitados electrónicamente, sirviendo mejor a los principios de eficacia y eficiencia, reforzando las obligaciones de transparencia y garantías de los interesados (ESPAÑA, 2015a).

Estos movimientos demuestran que el gobierno español está dirigiendo esfuerzos para la consolidación y maduración del proceso democrático.

El propio Plan de Transformación Digital de la Administración General del Estado y sus Organismos Públicos (ESPAÑA, 2015b), que constituye el marco estratégico global para avanzar en la transformación de la Administración Pública, se elaboró teniendo en cuenta las leyes aquí citadas.

El documento establece los objetivos e iniciativas específicas para conformar una administración ágil, eficaz, con un aprovechamiento óptimo de sus recursos y más cercana, para que los servicios públicos se adapten mejor a las necesidades reales de la sociedad y de una economía competitiva y emprendedora. Presenta como principios rectores: redefinir y orientar los servicios por el lado del usuario; asegurar una visión holística de las necesidades y acciones; sumar esfuerzos para fomentar la colaboración inter e intra administrativa y gestionar alianzas; permitir a los ciudadanos la monitorización y el control de la actividad de la administración (transparencia y la rendición de cuentas); y fomentar la innovación para impulsar el progreso y una mejor adaptación a los cambios (ESPAÑA, 2015b).

\section{METODOLOGÍA}

Considerando su importancia en el actual contexto político, económico y social, elegimos la Ley española 19/2013 como objeto de investigación para este artículo. Con el fin de realizar un estudio longitudinal con el objetivo de verificar en qué medida la Ley está siendo debidamente cumplida por los entes administrativos vía canales institucionales, sus aspectos más importantes referentes a la exigencia de publicidad activa en materia institucional, organizativa, de relevancia jurídica, económica, presupuestaria y estadística, y el reconocimiento del derecho de acceso a la información pública y la creación de un procedimiento para el ejercicio del mismo, fueron redactadas preguntas, estructuradas bajo la forma de un cuestionario creado a partir de la herramienta Survey Monkey. 
El cuestionario fue aplicado tres veces, por evaluadores distintos, con edad entre 18 y 24 años, alumnos del curso de Grado en Derecho de la Universidad de Zaragoza, en España, por ocasión de la oferta de las asignaturas de Derecho y Ética (en 9/2013 y 10/2015) y Sociología Jurídica (2/2015).

Después de recibir instrucciones sobre cómo proceder a la evaluación, el enlace para acceder al cuestionario fue puesto a disposición de los alumnos a través de Internet. Una cartilla fue creada con el fin de unificar la comprensión acerca de las variables a analizar, a efectos de que, con independencia del evaluador, los elementos buscados en los sitios fueran los mismos.

Por entender que la transparencia y el acceso a la información son derechos de los ciudadanos, el sitio del Defensor del Pueblo fue intencionalmente elegido para este estudio por ser la Institución encargada de defender los derechos fundamentales y las libertades públicas de esos actores mediante la supervisión de la actividad de las administraciones públicas. En teoría, si esa es la institución que va a cobrar a los demás, se espera que sea la que sirva de ejemplo a ellos.

Esa institución, que se considera transparente, hizo pública en el 14 de enero de 2013, por medio de su sitio web, la sección de transparencia, y durante todo ese año, 2014, 2015 y 2016 fueron añadidos contenidos considerados significativos para el conocimiento general, más allá de los exigidos por la citada Ley 19/2013.

Con el fin de verificar si el Defensor del Pueblo hace justicia a ese "título", la sección que sigue presenta el análisis de los datos y la síntesis de los resultados advindos de la comparación realizada entre los estudios efectuados en las tres oportunidades.

\section{ANÁLISIS DE DATOS}

Con el fin de cubrir todos los aspectos principales de la Ley 19/2013, 30 preguntas fueron estructuradas y los resultados se presentan a continuación.

Aunque la Ley de Transparencia, Acceso a la Información Pública y Buen Gobierno está presente en el ordenamiento jurídico español hace poco más de tres años, los procedimientos por ella establecidos, con el fin de garantizar el acceso a la información, aún no son integralmente atendidos por la Institución Defensor del Pueblo. A pesar de ello, se puede decir que, en general, se observó una mejora en la estructuración del sitio entre 2013 y 2015 para atender a las normativas dispuestas por la ley.

Desde 2013, el sitio presenta su propio Proyecto de Regulación sobre Transparencia, Acceso a la Información Pública y Buen Gobierno. El proyecto ha sido lanzado contiendo: a) las instrucciones sobre cómo solicitar el acceso a la información; y b) información sobre la autoridad responsable de la vigilancia por la 
aplicación de la normativa de Acceso a la Información.

Apenas en la tercera evaluación fueron encontradas informaciones relativas a: a) los límites al derecho de acceso (casos en los que la información está restringida o es confidencial y no puede ser ofrecida a los ciudadanos); b) los mecanismos de apelación en el caso de que una solicitud de información fuera negada (procedimientos que deben ser hechos en el caso de que una solicitud de información sea rechazada); y c) información acerca del plazo máximo de respuesta a una solicitud de información.

Para facilitar el ejercicio del derecho de acceso a la información pública, la Ley 19/2013 establece un mes como plazo de respuesta y sugiere la creación de unidades de información con la intención de aclarar a quienes presentar las solicitudes, así como el cuerpo competente para el procesamiento (ESPAÑA, 2013).

Acerca de la información institucional, organizativa y de planificación, la Ley 19/2013 hace referencia a la obligación de dar publicidad a:

a. Planes y programas anuales y plurianuales en los que se fijen objetivos concretos, así como las actividades, medios y tiempo previsto para su consecución;

b. Las directrices, instrucciones, acuerdos, circulares o respuestas a consultas planteadas por los particulares u otros órganos en la medida que supongan una interpretación del Derecho o tengan efectos jurídicos;

c. Los informes sobre Anteproyectos de Ley u otra normativa cuya iniciativa le corresponda a la entidad responsable, cuando se le hayan solicitado dictámenes por los órganos consultivos correspondientes;

d. Los proyectos de reforma normativa cuya iniciativa les corresponda;

e. Los documentos que, conforme a la legislación sectorial vigente, deban ser sometidos a un período de información pública durante su tramitación;

f. Las memorias e informes que conformen los expedientes de elaboración de los textos normativos, en particular, la memoria del análisis de impacto normativo regulada por el Real Decreto 1083/2009, de 3 de julio.

En 2013, el sitio presentaba esos documentos de manera incompleta. Solamente a partir de la segunda evaluación, en $2 / 2015$, es que todos los elementos citados fueron encontrados.

La publicación de la información institucional, organizativa y de planificación permite a los ciudadanos conocer a sus representantes y sus competencias, saber cómo ellos planean asignar el dinero público, cuáles son sus prioridades y metas de actuación.

La falta de claridad en lo que se refiere a la actividad de la entidad, las competencias de los representantes y sus propuestas, hace que el ciudadano no sepa exactamente lo que corresponde al ente gubernamental ni lo que van a hacer y a quién cobrar respuestas. 
La publicación de un organigrama actualizado con el diseño de la estructura organizativa también es mencionada por la Ley, una vez que permite que los ciudadanos sepan quiénes son los representantes de la institución, cómo están conectados y jerárquicamente organizados.

Desde 2013, está disponible en el sitio un organigrama haciendo referencia a toda la estructura de la Institución del Defensor del Pueblo, con identificación de los responsables de los diferentes órganos, incluyendo perfil y trayectoria profesional.

Acerca de la información económica, presupuestaria y estadística, la Ley 19/2013 menciona la obligación de dar publicidad a:

a) Todos los contratos formalizados, con indicación del objeto, el importe de licitación y de adjudicación, el procedimiento utilizado para su celebración, los instrumentos a través de los que, en su caso, se ha publicitado, el número de licitadores participantes en el procedimiento y la identidad del adjudicatario, así como las modificaciones del contrato. En 2013 y en 10/2015, los contratos han sido encontrados conteniendo todas las indicaciones citadas, pero en 2/2015, algunos quedaban incompletos;

b) La relación de los convenios suscritos, con mención de las partes firmantes, su objeto, plazo de duración, modificaciones realizadas, obligados a la realización de las prestaciones y, en su caso, las obligaciones económicas convenidas. Desde 2013, los convenios presentan problemas en su divulgación, pues no contienen todos los elementos citados. A veces, les faltan las obligaciones económicas convenidas, y otras el plazo de duración;

c) Las subvenciones y ayudas públicas concedidas con indicación de su importe, objetivo o finalidad y beneficiarios. En la última evaluación, las subvenciones presentaban apenas parte de las indicaciones mencionadas. En las dos anteriores, nada constaba a respecto de este tema;

d) Los presupuestos, con descripción de las principales partidas presupuestarias e información actualizada sobre su estado de ejecución. Aunque en 2013 ha sido diferente (todos los presupuestos disponibles estaban completos), el status de las informaciones sobre los presupuestos se mantuvo el mismo en 2015: los documentos son presentados de manera incompleta y no contienen todos los elementos citados;

e) Las cuentas anuales que deban rendirse y los informes de auditoría de cuentas y de fiscalización por parte de los órganos de control externo que sobre ellos se emitan;

f) Las resoluciones dictadas por la Oficina de Buen Gobierno y Conflictos de Intereses u organismo autonómico equivalente sobre la compatibilidad de las actividades privadas a realizar por los cargos mencionados en el artículo 3 de la Ley 5/2006, de 10 de abril, de conflictos de intereses de los miembros del Gobierno y de los Altos Cargos de la Administración General del Estado o en la legislación 
autonómica correspondiente. En las dos primeras evaluaciones hechas en el sitio del Defensor del Pueblo, ninguna información al respecto de las cuentas anuales y las resoluciones había sido encontrada. En la tercera oportunidad, parte de los documentos estaban disponibles, aunque no totalmente en conformidad con lo que la Ley obliga;

g) Las retribuciones percibidas anualmente por los altos cargos y máximos responsables de las entidades. En 2013, las retribuciones percibidas por los altos cargos constaban en los documentos divulgados en el sitio, pero a partir de 2015 ellas no fueron encontradas.

La publicación de información económica y presupuestaria del Estado permite a los ciudadanos acompañar y fiscalizar la aplicación del dinero público. El objetivo es dar mayor transparencia a los actos administrativos y contribuir al aumento del control social, de la gestión democrática y del combate a la corrupción.

En ese sentido, es fundamental que las informaciones fiscales y administrativas se presenten en un formato que sea comprensible por todos los públicos. Se debe tener en cuenta que muchos de los ciudadanos no conocen el tema con propiedad, de modo que la disposición de datos superficiales y tablas genéricas sin ningún análisis y confrontación de valores acaba por no agregar información, impidiendo su juicio y aplicación en los procesos de control social y toma de decisión.

Rocha (2012) resalta que la efectividad de la participación ciudadana está condicionada al acceso de informaciones precisas y confiables, que permitan la construcción de un marco referencial de la actuación del gobierno. A partir de ahí, el ciudadano puede exigir de los representantes explicaciones sobre sus acciones, cambios en la forma de actuar o incluso ofrecer sugerencias para cambios en los objetivos de las políticas públicas.

Una reflexión sobre la forma (escrita y visual) de presentar los informes que dan base para el análisis del ejercicio de la transparencia por las entidades gubernamentales es relevante e imprescindible.

h) La información estadística necesaria para valorar el grado de cumplimiento y calidad de los servicios públicos que sean de su competencia.

Desde 2013, es posible encontrar en el sitio del Defensor del Pueblo métricas que auxilian a los interesados en evaluar el grado de cumplimento y la calidad de los servicios públicos ofertados a los ciudadanos.

Con el fin de comprobar si la información disponible en el sitio es comprensible, de acceso fácil, gratuito y respecta a las personas con discapacidad en una modalidad suministrada por medios o en formatos adecuados de manera que resulten accesibles y comprensibles, conforme al principio de accesibilidad universal y diseño para todos, algunas preguntas fueran hechas.

Acerca del uso de medios para facilitar el acceso a la información por personas con discapacidad, el portal 
ofrece:

a) consejos de atajos para la navegación (consejos para un rápido acceso a ciertos elementos del portal, por lo general el menú principal, el contenido y la herramienta de búsqueda. Estos consejos deben estar disponibles en la barra de accesibilidad y en la página de accesibilidad del portal);

b) es posible ver el portal en otro idioma (el portal se puede acceder en otros idiomas diferentes a los de la/s lengua/s oficial/es del país);

c) Desde la primera evaluación han dado atención a estos quesitos.

d) servicios estructurados por perfiles, eventos o grupos de destinatarios (servicios agrupados y clasificados en función del perfil del usuario), acontecimientos de la vida (nacimiento, infancia y adolescencia, adultez, vejez y muerte) o grupos destinatarios (ciudadanos, empresas o gobierno);

e) opciones para redimensionar el tamaño del texto a través de iconos que permiten aumentar o disminuir el tamaño de fuente del texto disponible en la página web (ello está simbolizado normalmente por las letras $\mathrm{A}, \mathrm{A}+, \mathrm{A}-)$;

f) texto explicativo para los vídeos;

g) texto explicativo para las imágenes;

h) texto explicativo para los audios.

La estructuración de los servicios en función del perfil del público fue observada a partir de la última evaluación, hecha en 10/2015, así como la opción para redimensionar el tamaño del texto, el texto explicativo para los vídeos, imágenes y audios para algunos de los contenidos disponibles en el sitio.

a) opciones para cambiar el contraste, haciendo que un objeto sea distinguible de los demás y del fondo.

Esta opción aún no está disponible en el sitio del Defensor del Pueblo.

La mala operación de la usabilidad y de la accesibilidad puede dificultar el acceso a la información e incluso inviabilizar la participación ciudadana en los procesos decisorios, lo que puede conducir a la baja aceptación de esos canales por la sociedad.

Se entiende que, cuanto más simple y facilitado sea el acceso a los contenidos, menos errores el ciudadano podrá cometer, y más posibilidades habrá de localizar lo que se busca, aumentando la posibilidad e incluso el interés en participar y a elegir el sitio como canal de comunicación con el gobierno.

\section{SÍNTESIS DE LOS RESULTADOS}


Como se mencionó anteriormente, 30 variables (una por pregunta) fueron investigadas en el sitio institucional del Defensor del Pueblo. Si un punto fuera asignado a cada elemento encontrado por completo en el sitio, 0,5 para los encontrados parcialmente y 0 para los no encontrados, el escenario establecido sería el demostrado en la Figura 1.

Aunque el sitio del Defensor del Pueblo no atienda a todos los requisitos investigados, se percibe una constante mejora a la atención de la Ley 19/2013 desde la primera evaluación.

En 2013, año en que la Ley entró en vigor, menos de la mitad de los elementos buscados fueron encontrados (40\%). Con el paso del tiempo, probablemente en función de la mayor comprensión a respecto de la norma y de su importancia para el ejercicio de la transparencia y, consecuentemente, de la participación y control social, nuevos aspectos fueran siendo incorporados, de modo que, en la tercera evaluación (hecha el 10/2015), un $80 \%$ de los elementos estaban presentes.

\section{Figura 1: Desempeño del sitio Defensor del Pueblo en las tres evaluaciones}

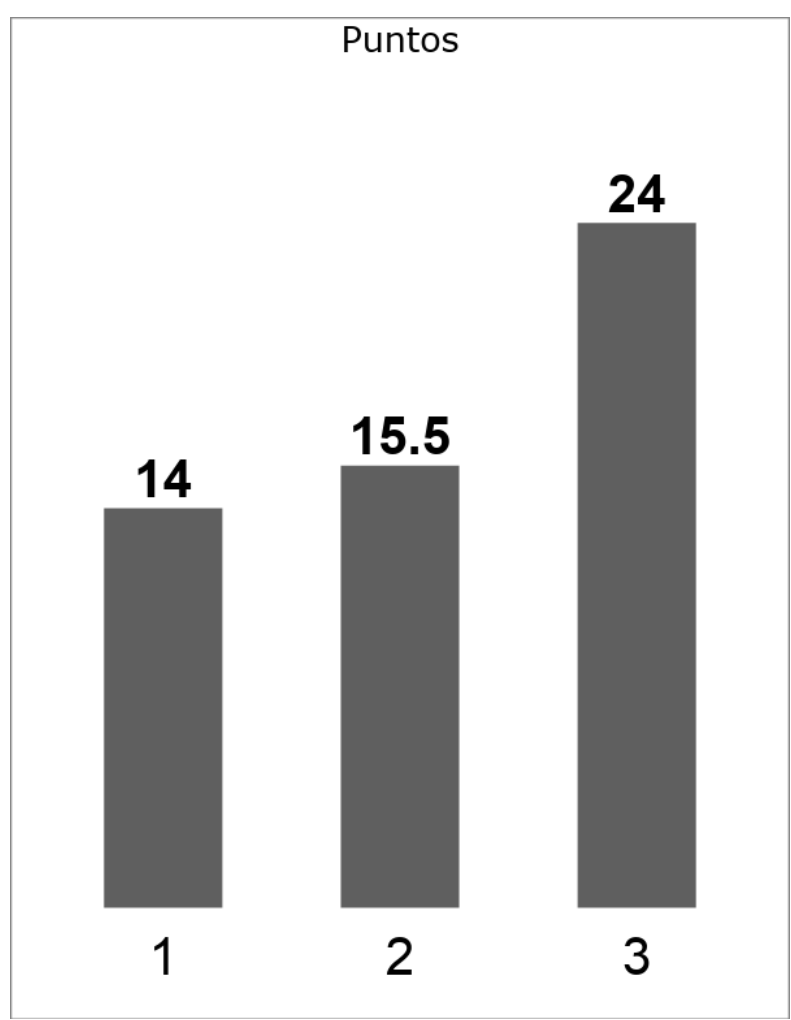

Esta constatación va al encuentro de lo afirmado por la institución en su sitio web, que dice que, poco a poco, nuevas informaciones se fueron poniendo a la disposición de los ciudadanos con el objetivo de dar transparencia a las acciones administrativas.

Comparando los principales aspectos evaluados en las tres oportunidades (proyecto; información 
institucional, organizativa y de planificación; información económica, presupuestaria y estadística; usabilidad y accesibilidad), se percibe que, proporcionalmente presentan más mejoras el aspecto de proyecto (50\% en la primera y segunda evaluaciones x 100\% en la tercera), seguido por la información institucional, organizativa y de planificación (56,25\% en la primera x 100\% en la segunda y tercera) y por la usabilidad y accesibilidad (25\% en la primera y segunda evaluaciones x 68,75\% en la tercera), según la Tabla 1 .

\section{Tabla 1: Aspectos evaluados en el sitio institucional del Defensor del Pueblo}

\begin{tabular}{|c|c|c|c|}
\hline Aspectos evaluados & $\begin{array}{c}1^{\text {a }} \\
\text { Evaluación }\end{array}$ & 2a Evaluación & 3a Evaluación \\
\hline Proyecto & $50 \%$ & $50 \%$ & $100 \%$ \\
\hline $\begin{array}{c}\text { Información institucional, organizativa y de } \\
\text { planificación }\end{array}$ & $56,25 \%$ & $100 \%$ & $100 \%$ \\
\hline $\begin{array}{c}\text { Información económica, presupuestaria y estadística } \\
\text { Usabilidad y accesibilidad }\end{array}$ & $56,25 \%$ & $31,25 \%$ & $56,25 \%$ \\
\hline
\end{tabular}

Es interesante y preocupante notar que las informaciones económicas, presupuestarias y estadísticas se mantuvieron incompletas desde la primera evaluación, presentando aún una ligera disminución en 2/2015.

No se sabe, todavía, por qué la institución ha dedicado poca atención a este aspecto, dada su importancia frente al ejercicio del control social y de la transparencia pública. Puede que estén buscando la mejor manera de presentar la información, según los distintos niveles de conocimiento del público, o que no tengan interés en ponerla a disposición de los ciudadanos. Cabe una investigación más profunda en este caso.

\section{CONSIDERACIONES FINALES Y TRABAJOS FUTUROS}

Este artículo buscó realizar un estudio longitudinal para verificar si las prerrogativas de la Ley española 19/2013, de 9 de diciembre, de Transparencia, Acceso a la Información Pública y Buen Gobierno están siendo atendidas por la institución del Defensor del Pueblo.

Es posible concluir que, aunque el sitio del Defensor del Pueblo no atienda a todos los requisitos investigados, se percibe una constante mejora a la atención de la Ley desde la primera evaluación. En 2013, año en que la Ley entró en vigor, menos de la mitad de los elementos buscados fueron encontrados. Con el paso del tiempo, probablemente en función de la mayor comprensión a respecto de la norma y de su importancia para el ejercicio de la transparencia y, consecuentemente, de la participación y del control social, nuevos aspectos fueran incorporados, de modo que, en la tercera evaluación, un $80 \%$ de los elementos estaban presentes. 
Comparando los principales aspectos evaluados en las tres oportunidades, se percibe que, proporcionalmente, presentan más mejoras el aspecto de proyecto, seguido por la información institucional, organizativa y de planificación, y por la usabilidad y accesibilidad. Es interesante y preocupante notar que las informaciones económicas, presupuestarias y estadísticas se mantuvieron incompletas desde la primera evaluación.

Este es un aspecto muy importante en el contexto de fortalecer la acción de los ciudadanos. Sin embargo, aparece poco tratado por el sitio del Defensor del Pueblo, lo que merece una investigación en un trabajo futuro.

\title{
TRANSPARENCY AND ACCESS TO INFORMATION ON THE DEFENSOR DEL PUEBLO WEBSITE: A LONGITUDINAL STUDY
}

\begin{abstract}
Government websites should be increasingly open, welcoming and receptive to social control, in order to enable citizen participation in the decision-making process. Thus, they must present specific content to their target audience and allow knowledge construction and sharing. Since access and publicity of information are essential conditions for the establishment of the Democratic State of Law, this article seeks to investigate whether the prerogatives of Spanish Law 19/2013, on Transparency, Access to Public Information and Good Government, are being met by the Defensor del Pueblo institution. Its most important aspects, related to the active publicity requirement, organizational, legal, economic, budgetary and statistical matters, and the recognition of the right of access to public information and the creation of a procedure that allows its exercise, were translated into 30 questions, structured in the form of a questionnaire created from the Survey Monkey tool. The evaluation shows that the procedures established by the Law, in order to guarantee access to information, are not yet fully addressed by the Defensor del Pueblo Institution.
\end{abstract}

Keywords: Longitudinal study; Government websites; Transparency; Access to information; Democratic State of Law.

\section{REFERENCIAS}

AL-MUDIMIGH, Abdullah S.; ULLAH, Zahid. Effective Implementation of Portals: Best Practice Model. International Journal of Business and Management, Toronto, v. 6, n. 2, p. 46-51, 2011.

AMORIM, Paula K. D. F. Democracia e internet: a transparência de gestão nos portais eletrônicos das capitais brasileiras. 2012. 348 p. Tese (Doutorado) - Programa de Pós-Graduação em Comunicação e Cultura Contemporâneas, Faculdade de Comunicação, Universidade Federal da Bahia. Salvador.

BOBBIO, Norberto. O futuro da democracia. 8. ed. São Paulo: Paz e Terra, 2002. 
CORONEL, Sheila S. Measuring Openness: A survey of transparency ratings and the prospects for a global index. Open Society Foundations, 2012. Disponível em: <http://www.freedominfo.org/2012/10/measuringopenness-a-survey-of-transparency-ratings-and-the-prospects-for-a-global-index/>. Consultado el: 7 ene. 2013.

ESPAÑA. Ley 37/2007, de 16 de noviembre, sobre reutilización de la información del sector público. BOE, Jefatura del Estado, Madrid, 17 nov. 2007. Sección I, n. 276, p. 47160-47165. Disponible en: <https://www.boe.es/buscar/pdf/2007/BOE-A-2007-19814-consolidado.pdf>. Consultado el: 20 ago. 2015.

Ley 19/2013, de 9 de diciembre, de transparencia, acceso a la información pública y buen gobierno. Boletín Oficial del Estado, Jefatura del Estado, Madrid, 10 dic. 2013. Sección I, n. 295, p. 97922-97952. Disponible en: <https://www.boe.es/buscar/pdf/2013/BOE-A-2013-12887-consolidado.pdf>. Consultado el: 20 ago. 2015.

Ley 39/2015, de $1^{\circ}$ de octubre, del Procedimiento Administrativo Común de las Administraciones Públicas. Boletín Oficial del Estado, Jefatura del Estado, Madrid, 2 oct. $2015 a$. Sección I, n. 236. BOE-A-201510565. Disponible en: <https://www.boe.es/buscar/pdf/2015/BOE-A-2015-10565-consolidado.pdf>. Consultado el: 3 mayo 2017.

Ministerio de Hacienda y Administraciones Públicas. Dirección de Tecnologías de la Información y las Comunicaciones (DTIC). Plan de Transformación Digital de la Administración General del Estado y sus Organismos Públicos (Estrategia TIC 2015 - 2020). (2015b) Disponible en: $<$ http://administracionelectronica.gob.es/pae_Home/pae_Estrategias/Estrategia-TIC-

AGE.html\#.WQN_BmW0mOM>. Consultado el: 15 feb. 2017.

HOLZNER, Burkart; HOLZNER, Leslie. Transparency on global change: the vanguard of the open society. Pittsburgh: University of Pittsburgh Press, 2006.

LINDVALL, Mikael; RUS, Ioana; SINHA, Sachin S. Software systems support for knowledge management. Journal of Knowledge Management, v. 7, n. 5, p. 137-150, 2003.

MARQUES, Francisco P. J. A. Dimensões da Ciberdemocracia: conceitos e experiências fundamentais. 2004. 207 p. Dissertação (Mestrado) - Programa de Pós-Graduação em Comunicação e Cultura Contemporâneas, Faculdade de Comunicação, Universidade Federal da Bahia. Salvador.

OECD. Focus on Citizens: public engagement for better policy and services. OECD: Paris, 2009.

SANTOS, Paloma M. Framework de apoio à democracia eletrônica em portais de governo com base nas práticas de gestão do conhecimento. 2014. 430 p. Tese (Doutorado) - Programa de Pós-Graduação em Engenharia e Gestão do Conhecimento, Centro Tecnológico, Universidade Federal de Santa Catarina. Florianópolis, 2014.

SILVA, Sivaldo P. Estado, democracia e internet: requisitos democráticos e dimensões analíticas para a interface digital do Estado. 2009. 424 p. Tese (Doutorado) - Programa de Pós-Graduação em Comunicação e Cultura Contemporâneas, Faculdade de Comunicação, Universidade Federal da Bahia. Salvador.

TORRES, Marcelo D. F. Estado, democracia e administração pública no Brasil. Rio de Janeiro: Editora FGV, 2004. 
Trabalho enviado em 04 de dezembro de 2017.

Aceito em 14 de março de 2018. 